Astronomical Polarimetry: Current Status and Future Directions

ASP Conference Series, Vol. 343, 2005

Adamson, Aspin, Davis, and Fujiyoshi

\title{
Tracing the Magnetic Field in Molecular Clouds
}

\author{
Martin Houde, Ruisheng Peng, and Hiroshige Yoshida \\ Caltech Submillimeter Observatory, 111 Nowelo Street, Hilo, HI 96720, \\ USA
}

Roger H. Hildebrand

Department of Astronomy and Astrophysics, Enrico Fermi Institute, and Department of Physics, University of Chicago, Chicago, IL 60637, USA

C. Darren Dowell

California Institute of Technology, Pasadena, CA 91125, and Jet Propulsion Laboratory, 4800 Oak Grove Drive, Pasadena, CA 91109, $U S A$

Thomas G. Phillips

California Institute of Technology, Pasadena, CA 91125, USA

Jessie L. Dotson

NASA Ames Research Center, Moffet Field, CA 94035, USA

Pierre Bastien

Département de Physique, Université de Montréal, Montréal, Québec H3C 3J7, Canada

John E. Vaillancourt

Physics Department, University of Wisconsin, 1150 University Avenue, Madison, WI 53706, USA

\begin{abstract}
We discuss how the combination of polarimetry and ion-to-neutral molecular line width ratio measurements permits the determination of the orientation of the magnetic field in the weakly ionized parts of molecular clouds. Polarimetry measurements give the field orientation in the plane of the sky, and the ion-to-neutral molecular line width ratio determines the angle between the magnetic field and the line of sight. We present the results obtained with this technique on the M17 and Orion A star-forming regions using Hertz $350 \mu \mathrm{m}$ polarimetry maps and $\mathrm{HCO}^{+}$-to-HCN molecular line width ratios to provide the first view of the spatial orientation of the magnetic field these molecular clouds.
\end{abstract}

\section{Introduction}

The orientation of the magnetic field vector at any given point in a molecular cloud can be specified with two parameters: $\alpha$, its inclination angle to the line of 
sight, and $\beta$, its projection angle on the plane of the sky (eastward from north). Until recently, of these two angles, only $\beta$ could be measured. At submillimeter wavelengths, this can be accomplished, for example, with polarization measurements of the continuum radiation emanating from elongated dust grains that are aligned by the local magnetic field (Davis \& Greenstein 1952). The angle $\beta$ is thus obtained from the angle of the polarization vector since the projection of the magnetic field vector in the plane of the sky is oriented at right angles to the polarization vector (Hildebrand 1988).

In this paper, we will discuss how it is possible to determine the inclination angle $\alpha$ through the combination of spectroscopy and polarimetry data. We will show that this is made possible with the realization (Houde et al. 2000a,b) that the magnetic field leaves its imprint on the line profiles of molecular ions. Indeed, the ion-to-neutral line width ratio, calculated from the line profiles of coexistent ion and neutral molecular species, is the fundamental parameter that, when combined with polarimetry data, holds the key to the determination of the inclination angle of the magnetic field.

We will summarize the technique and the first results obtained for the M17 molecular cloud and Orion A complex. We use $\mathrm{HCO}^{+}$and $\mathrm{HCN}$ spectroscopic data, and extensive $350 \mu \mathrm{m}$ continuum and polarimetry maps obtained with the SHARC II camera (Dowell et al. 2003) and the Hertz polarimeter (Dowell et al. 1998) at the Caltech Submillimeter Observatory (CSO). These results were previously published in Houde et al. $(2002,2004)$.

\section{The Effect of the Magnetic Field on the Line Profiles of Molecular Ions}

Houde et al. (2000a,b) have discussed the effect that the magnetic field can have on molecular ions in the weakly ionized and turbulent regions of molecular clouds. They have shown that, because of the fact that an ion is trapped and undergoes gyromagnetic motions when in the presence of a sufficiently strong magnetic field, its translational motions are restricted to directions that are parallel to the orientation of the local field. It cannot, therefore, fully take part in the same turbulent regime that coexistent neutral species are subjected to. One direct consequence is the realization that the width of the line profiles of molecular ions should, in general, be less than that of coexistent neutral molecular species in cases where the orientation of the magnetic field is not, on average, aligned with the flows that characterize the turbulent motions of the neutral component of the gas. This fact has been verified experimentally in numerous molecular clouds through the observations and comparisons of coexistent neutral and ion molecular species spectra (see Houde et al. 2000a,b).

\section{The Evaluation of the Inclination Angle $\alpha$}

Since, as was stated before, the motions of molecular ions are restricted to directions parallel to that of the magnetic field (neglecting gyrations), it should not be surprising if the width of their line profiles were to be a function of the orientation of the field to the line of sight. It can, in fact, be shown that this is so, at least when considering reasonably general models of neutral flow 
configurations (see Figure 2 of Houde et al. 2002, and the discussion that pertains to it). Moreover, it is also the case that the ion-to-neutral line width ratio, as calculated from the line profiles of coexistent ion and neutral molecular species in the context of these models, is a well-behaved function of the inclination angle. It is maximum at approximately unity when the field is aligned with the line of sight $(\alpha=0)$, and decreases monotonically to a minimum $(\simeq 0.26$ for $\left.\mathrm{HCO}^{+}\right)$when the magnetic field lies in the plane of the sky $(\alpha=\pi / 2)$. There is, however, an ambiguity in selecting which model of neutral flows is best suited to a given region. It is necessary to discriminate between the different alternatives in order to succeed in calculating $\alpha$ from the ion-to-neutral line width ratio.

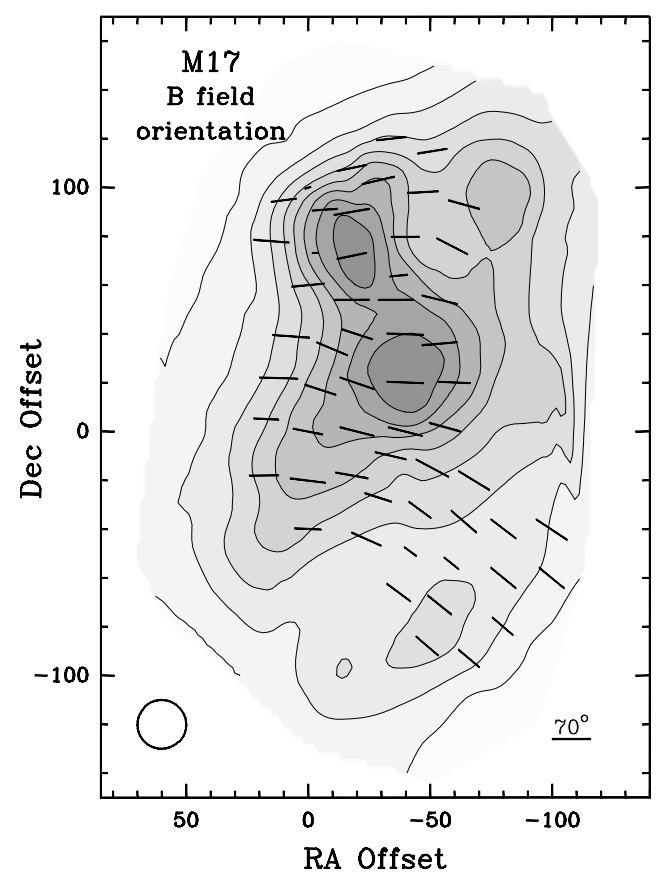

Figure 1. Orientation of the magnetic field in M17. The orientation of the projection of the magnetic field in the plane of the sky is shown by the vectors and the viewing angle is given by the length of the vectors (using the scale shown in the bottom right corner). The contours and the grey scale delineate the total continuum flux. The Hertz beam width $\left(\simeq 20^{\prime \prime}\right)$ is shown in the lower left corner.

This is accomplished by using another observable parameter that is also a function of the orientation of the magnetic field relative to the line of sight. This parameter is the polarization level $P$ that is measured, for example, from dust continuum emission at submillimeter wavelengths. More precisely, the polarization level $P$, detected by an observer studying a given region where the field is oriented with a viewing angle $\alpha$, can be expressed as

$$
P=P_{\max } \sin ^{2}(\alpha)
$$




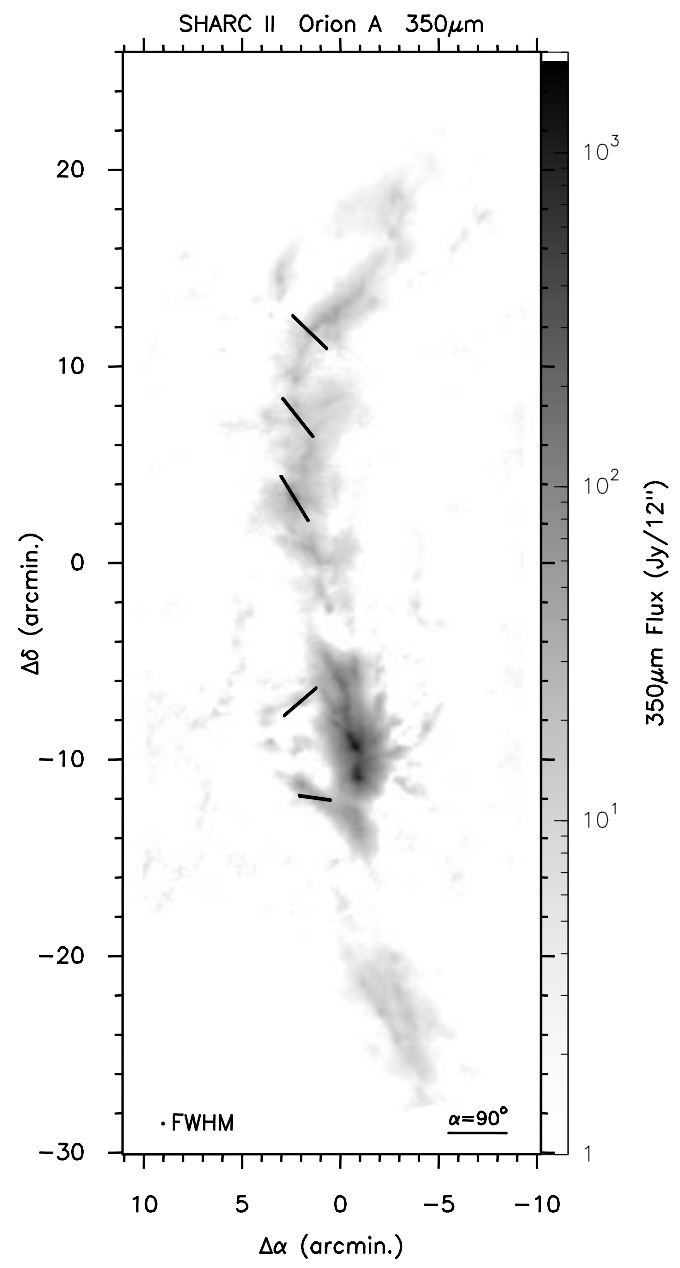

Figure 2. $350 \mu \mathrm{m}$ continuum map of the Orion A region obtained with SHARC II. The orientation of the magnetic field is indicated at five positions along the ISF on the map. The projection of the magnetic field in the plane of the sky is also shown by the orientation of the accompanying vectors and the inclination angle is given by the length of the vectors (using the scale shown in the bottom right corner). The SHARC II beam width is shown in the lower left corner $\left(\simeq 12^{\prime \prime}\right)$.

where $P_{\max }$ is the maximum polarization level that can be detected, i.e. when the field lies in the plane of the sky $(\alpha=\pi / 2)$. Equation (1) can be used in conjunction with the relevant equation for the ion-to-neutral line width ratio in order to express the latter as a function of the normalized polarization level $P / P_{\max }$. By carefully taking into account the well-known depolarization effect, characterized by a systematic decrease in the polarization level with increasing continuum flux, and assigning a suitable value for $P_{\max }$, it then becomes possible to use polarization data to constrain the neutral flow model and, in effect, calibrate the ion-to-neutral line width ratio. 


\subsection{The M17 Molecular Cloud and the Orion A Complex}

The previously summarized technique was used to measure the orientation of the magnetic field in the M17 molecular cloud (Houde et al. 2002) and the Orion A complex (Houde et al. 2004). These results were acquired using $350 \mu \mathrm{m}$ polarimetry and spectroscopic ( $\mathrm{HCN}$ and $\mathrm{HCO}^{+}$in the $J \rightarrow 4-3$ transition) maps obtained using the Hertz polarimeter (Dowell et al. 1998) and the CSO 300-400 GHz receiver, respectively. We also benefited from an extensive $350 \mu \mathrm{m}$ SHARC II map (Dowell et al. 2003) for the Orion A analysis.

A detailed view of the orientation of the magnetic field in M17 is shown in Figure 1 (see the caption) where $\alpha$ is seen not to exceed $\approx 60^{\circ}$. This value was combined with Zeeman measurements from Brogan \& Troland (2001) by Houde et al. (2002) to calculate a magnitude of $\approx 1.5 \mathrm{mG}$ for the magnetic field in this molecular cloud.

Average orientations of the magnetic field were calculated for Orion A. It was found that the orientation of the magnetic field is relatively stable in OMC-2 and OMC-3 where $\alpha \sim 75^{\circ}$ and $\beta \sim 40^{\circ}$, while it exhibits significant re-orientations in OMC-1 (see Table 1 of Houde et al. 2004). We can then infer that the field seems to be relatively unaffected by the presence of the concentrations of lower mass that characterize the OMC-2 and OMC-3 regions, while it appears to be strongly perturbed in and around the massive OMC-1 complex. We present the Orion A SHARC II map with the average orientation of the magnetic field at five different positions along the integral-shaped filament (ISF) in Figure 2.

Acknowledgments. The Caltech Submillimeter Observatory is funded by the NSF through contract AST 9980846 and the observations made with Hertz were supported by NSF Grants AST 9987441 and AST 0204886.

\section{References}

Brogan, C. L., \& Troland, T. H. 2001, ApJ, 560, 821

Davis, L. M. \& Greenstein, J. L. 1952, ApJ, 114, 206

Dowell, C. D., Hildebrand, R. H., Schleuning, D. A., Vaillancourt, J. E., Dotson, J. L., Novak, G., Renbarger, T., \& Houde, M. 1998, ApJ, 504, 588

Dowell, C. D., Allen, C. A., Babu, S., Freund, M. M., Gardner, M., Groseth, J., Jhabvala, M., Kovacs, A., Lis, D. C., Moseley Jr., S. H., Phillips, T. G., Silverberg, R., Voellmer, G., \& Yoshida, H. 2003, in Proceedings of SPIE Vol. 4855, Millimeter and Submillimeter Detectors for Astronomy, eds. T. G. Phillips \& J. Zmuidzinas, (Bellingham, WA: SPIE), 73

Hildebrand, R. H. 1988, QJRAS, 29, 327

Houde, M., Bastien, P., Peng, R., Phillips, T. G., \& H. Yoshida 2000a, ApJ, 536, 857

Houde, M., Peng, R., Phillips, T. G., Bastien, P., \& Yoshida, H. 2000b, ApJ, 537, 245

Houde, M., Bastien, P., Dotson, J. L., Dowell, C. D., Hildebrand, R. H., Peng, R., Phillips, T. G., Vaillancourt, J. E., \& Yoshida, H. 2002, ApJ, 569, 803

Houde, M., Dowell, C. D., Hildebrand, R. H., Dotson, J. L., Vaillancourt, J. E., Phillips, T. G., Peng, R., \& Bastien, P. 2004, ApJ, 604, 717

\section{Discussion}

Crutcher: Your method could be tested by combining Chandrasekhar-Fermi estimates of B pos with Zeeman measurements of B los to determine the angle 
alpha. Do you think this would be possible? Can you estimate the uncertainty in your determinations of the angle alpha?

Houde: Yes, it could be tested this way. However, one has to keep in mind the uncertainties that remain with the Chandrasekhar-Fermi technique. More specifically, its tendency to overestimate the strength of the field. Nonetheless, this would be an interesting area of research. We provide uncertainties about the angle alpha in our analyses, and papers on the subject. However, the numbers given only take into account the corresponding uncertainties in the evaluation of spectral line widths. There is certainly an additional contribution to this from the selection of a single (neutral) turbulence model for the entire extent of a molecular clouds. This would probably increase the uncertainties by a few degrees in most cases. We discussed this issue in the first paper on the subject (Houde et al. 2002, ApJ, 569, 803).

Ward-Thompson: Do your data on the OMC filament rule out a helical magnetic field?

Houde: I don't believe it completely rules out a helical magnetic field in general. However, we do state in our paper on the Orion A star forming region (Houde et al. 2004, ApJ, 604, 717) that our results suggest that the orientation of the field in the northern part of the cloud may follow that of the magnetic field on a larger scale (extending well beyond the filament). If that statement were to be verified, then, the possibility of the presence of a helical magnetic field in the OMC filament would be greatly reduced. 